\title{
On the nonrelativistic limit of a semilinear field equation in a uniform and isotropic space
}

\author{
Makoto NAKAMURA *
}

\begin{abstract}
The nonrelativistic limit of a semilinear field equation is considered in a uniform and isotropic space. The scale-function of the space is constructed based on the Einstein equation. The Cauchy problem of the limit-equation is considered, and global and blow-up solutions are shown in Sobolev spaces. The role of spatial variance on the problem is studied, and some dissipative properties of the limit-equation are remarked.
\end{abstract}

Mathematics Subject Classification (2010): Primary 35Q55;Secondary 35L71,35Q75 Keywords: semilinear field equation, cosmological principle, nonrelativistic limit, Cauchy problem

\section{Introduction}

We consider a line element which has complex coefficients in a uniform and isotropic space. We use the Einstein equation to set its scale-function which describes the spatial variance. We consider a semilinear scalar-field equation in the space, and we take its nonrelativistic limit. We consider the Cauchy problem of the limit-equation, and we show global and blow-up solutions in Sobolev spaces. We first introduce the limit-equation in this section, and we show its derivation in the next section.

We denote the spatial dimension by $n \geq 1$, the Planck constant by $\hbar:=h / 2 \pi$, the mass by $m>0$. Let $\sigma \in \mathbb{R}, a_{0}>0, a_{1} \in \mathbb{R}$. We put $T_{0}:=\infty$ when $(1+\sigma) a_{1} \geq 0$, $T_{0}:=-2 a_{0} / n(1+\sigma) a_{1}(>0)$ when $(1+\sigma) a_{1}<0$. We define a scale-function $a(t)$ for $t \in\left[0, T_{0}\right)$ by

$$
a(t):= \begin{cases}a_{0}\left(1+\frac{n(1+\sigma) a_{1} t}{2 a_{0}}\right)^{2 / n(1+\sigma)} & \text { if } \sigma \neq-1, \\ a_{0} \exp \left(\frac{a_{1} t}{a_{0}}\right) & \text { if } \sigma=-1,\end{cases}
$$

${ }^{*}$ Faculty of Science, Yamagata University, Kojirakawa-machi 1-4-12, Yamagata 990-8560, JAPAN. E-mail: nakamura@sci.kj.yamagata-u.ac.jp 
where we note that $a_{0}=a(0)$ and $a_{1}=\partial_{t} a(0)$. We define a weight function $w(t):=$ $\left(a_{0} / a(t)\right)^{n / 2}$, and a change of variable $s=s(t):=\int_{0}^{t} a(\tau)^{-2} d \tau$. We put $S_{0}:=s\left(T_{0}\right)$. We use conventions $a(s):=a(t(s))$ and $w(s):=w(t(s))$ for $s \in\left[0, S_{0}\right)$ as far as there is no fear of confusion. A direct computation shows

$$
S_{0}= \begin{cases}\frac{2}{a_{0} a_{1}(4-n(1+\sigma))} & \text { if } a_{1}(4-n(1+\sigma))>0 \\ \infty & \text { if } a_{1}(4-n(1+\sigma)) \leq 0 .\end{cases}
$$

For $\lambda \in \mathbb{C}, 1 \leq p<\infty,-\pi / 2<\omega \leq \pi / 2,0 \leq \mu_{0}<n / 2$ and $0<S \leq S_{0}$, we consider the Cauchy problem given by

$$
\left\{\begin{array}{l} 
\pm i \frac{2 m}{\hbar} \partial_{s} u(s, x)+e^{-2 i \omega} \Delta u(s, x)-\lambda e^{-2 i \omega} a(s)^{2}\left(|u w|^{p-1} u\right)(s, x)=0, \\
u(0, \cdot)=u_{0}(\cdot) \in H^{\mu_{0}}\left(\mathbb{R}^{n}\right)
\end{array}\right.
$$

for $(s, x) \in[0, S) \times \mathbb{R}^{n}$, where $i:=\sqrt{-1}, \Delta:=\sum_{j=1}^{n} \partial^{2} / \partial x_{j}^{2}$, and $H^{\mu_{0}}\left(\mathbb{R}^{n}\right)$ denotes the Sobolev space of order $\mu_{0} \geq 0$. The double sign \pm is in same order throughout the paper. We say that $u$ is a global solution of (1.2) if it exists on $\left[0, S_{0}\right)$.

When the scale-function is a constant $a(\cdot)=a_{0}=1$, we have $w(\cdot)=1, s=t$ and $S_{0}=T_{0}=\infty$. The first equation in (1.2) corresponds to the Schrödinger equation

$$
\pm i \frac{2 m}{\hbar} \partial_{s} u(s, x)+\Delta u(s, x)-\lambda\left(|u|^{p-1} u\right)(s, x)=0
$$

when $\omega=0$, and to the parabolic equation

$$
\frac{2 m}{\hbar} \partial_{s} u(s, x)-\Delta u(s, x)+\lambda\left(|u|^{p-1} u\right)(s, x)=0
$$

when $\omega= \pm \pi / 4$. The complex Ginzburg-Landau equation

$$
\frac{2 m}{\hbar} \partial_{s} u(s, x)-\gamma \Delta u(s, x)-\lambda_{1} u(s, x)+\lambda_{2}\left(|u|^{2} u\right)(s, x)=0
$$

is also considered as the sum of the potentials with $p=1$ and $p=3$ for $0< \pm \omega<$ $\pi / 2$, where $\lambda_{1} \geq 0, \gamma \in \mathbb{C}$ with $\operatorname{Re} \gamma>0, \lambda_{2} \in \mathbb{C}$ with $\operatorname{Re} \lambda_{2}>0$. We refer to [16], 24], [31, (2.1)], [32], [33, (II.1) and the footnote in p.304] for the (generalized) complex Ginzburg-Landau equation. We note that the dimension of $\hbar / m$ in the SI units is $\mathrm{M}^{2} \mathrm{~S}^{-1}$ (M: meter, $\mathrm{S}$ : second), which is equivalent to the dimension of the thermal diffusivity $K_{1}$ of the heat equation $\partial_{s} u-K_{1} \Delta u=0$, and also to the dimension of the diffusion coefficient $K_{2}$ of the diffusion equation $\partial_{s} u-K_{2} \Delta u=0$.

Remark 1.1. Let us consider the diffusion equation in the space with a general scale-function $a(\cdot)>0$ which may be different from (1.1). We denote the diffusion constant by $K>0$. For infinitesimal $\Delta t>0$ and $\Delta r>0$ with $(\Delta r)^{2}=2 n K \Delta t$, we assume that the value of a function $\phi$ at $(t+\Delta t, x)$ is determined by the form

$$
\phi(t+\Delta t, x)=\frac{1}{n \omega_{n}}\left(\frac{a(t)}{\Delta r}\right)^{n-1} \int_{|y|=\Delta r / a(t)} \phi(t, x+y) d \sigma(y)+\int_{t}^{t+\Delta t} f(\tau, x) d \tau,
$$


where $\omega_{n}$ denotes the volume of the unit ball in $\mathbb{R}^{n}$, $d \sigma$ denotes the measure on the sphere, and $f$ denotes a force. By the mean value theorem and the divergence theorem, we have

$$
\partial_{t} \phi(t, x)=\frac{K}{a(t)^{2}} \Delta \phi(t, x)+f(t, x) .
$$

We put $f:=-n\left(\partial_{t} a\right) \phi / 2 a-J V_{1}(\phi)$ for a constant $J$ and a function $V_{1}$. We put the weight function $w(t):=(a(0) / a(t))^{n / 2}$, the change of variable $s=s(t):=$ $\int_{0}^{t} a(\tau)^{-2} d \tau$, and a new function $u(t, x):=\phi(t, x) w(t)^{-1}$. Then we have

$$
\partial_{s} u(s, x)-K \Delta u(s, x)+J a(s)^{2} w(s)^{-1} V_{1}(u w)(s, x)=0 .
$$

This diffusion equation corresponds to the first equation in (1.2), provided $\omega= \pm \pi / 4$, $J=K=\hbar / 2 m$, and $V_{1}(\phi)=\lambda|\phi|^{p-1} \phi$. We show a more general derivation of the first equation in (1.2) starting from a line element in the next section.

Remark 1.2. The first equation in (1.2) is rewritten as $\pm i \frac{2 m}{\hbar} \partial_{s} u(s, x)+e^{-2 i \omega} \Delta u(s, x)-\lambda e^{-2 i \omega} a_{0}^{n(p-1) / 2} a(s)^{2-n(p-1) / 2}\left(|u|^{p-1} u\right)(s, x)=0$.

We have $a(\cdot)^{2-n(p-1) / 2}=1$ when $p$ is the conformal power $p=1+4 / n$. This fact reminds us of the pseudo-conformal transform for (1.3) (see [35]). Indeed, the equation

$$
\pm 2 i \partial_{t} v(t, x)+e^{2 i \omega} \Delta v(t, x)-\bar{\lambda} e^{2 i \omega}\left(|v|^{p-1} v\right)(t, x)=0
$$

is transformed to

$\pm 2 i \partial_{s} u(s, x)+e^{-2 i \omega} \Delta u(s, x)-\lambda e^{-2 i \omega} s^{n(p-1) / 2-2} e^{ \pm(p-1)(\sin 2 \omega) x^{2} / 2 s}\left(|u|^{p-1} u\right)(s, x)=0$

by the transform $u(s, x)=s^{-n / 2} e^{ \pm i e^{2 i \omega} x^{2} / 2 s} \bar{v}(1 / s, x / s)$ with $t:=1 / s$ and $y:=x / s$. When $p=1+4 / n, \omega=0$ and $\lambda \in \mathbb{R}$, the equation is invariant.

It is well-known that the Schrödinger equation $i 2 m \partial_{t} u / \hbar+\Delta u-\lambda|u|^{p-1} u=0$ is derived from the Klein-Gordon equation $\partial_{t}^{2} \phi-c^{2} \Delta \phi+\left(m c^{2} / \hbar\right)^{2} \phi+c^{2} \lambda|\phi|^{p-1} \phi=0$ by the transform $\phi=u e^{-i m c^{2} t / \hbar}$ and the nonrelativistic limit $c \rightarrow \infty$. One of the aims of this paper is to show that the first equation in (1.2) which generalizes the Schrödinger equation and the parabolic equation is naturally derived from the nonrelativistic limit of the field equation (2.10), below. Moreover, we consider the spatial variance described by the scale-function $a(\cdot)$, which satisfies the Einstein equation with the cosmological constant in a uniform and isotropic space. The study of roles of the cosmological constant and the spatial variance is important to describe the history of the universe, especially, the inflation and the accelerating expansion of the universe. The scale-function (1.1) follows from the equation of state 
when we regard the cosmological constant as the dark energy (see (2.8), below). In this paper, we study the cosmological constant from the point of view of partial differential equations. We consider the Cauchy problem (1.2), and we show the well-posedness of the problem, global solutions and blow-up solutions. Especially, we remark that some dissipative properties appear by the spatial variance.

Let us consider the well-posedness of (1.2). For any real numbers $2 \leq q \leq \infty$ and $2 \leq r<\infty$, we say that the pair $(q, r)$ is admissible if it satisfies $1 / r+2 / n q=1 / 2$. For $\mu_{0} \geq 0$ and two admissible pairs $\left\{\left(q_{j}, r_{j}\right)\right\}_{j=1,2}$, we define a function space

$$
X^{\mu_{0}}([0, S)):=\left\{u \in C\left([0, S), H^{\mu_{0}}\left(\mathbb{R}^{n}\right)\right) ; \max _{\mu=0, \mu_{0}}\|u\|_{X^{\mu}([0, S))}<\infty\right\}
$$

where

$$
\|u\|_{X^{\mu}([0, S))}:= \begin{cases}\|u\|_{L^{\infty}\left((0, S), L^{2}\left(\mathbb{R}^{n}\right)\right) \cap \bigcap_{j=1,2} L^{q_{j}}\left((0, S), L^{r_{j}}\left(\mathbb{R}^{n}\right)\right)} & \text { if } \mu=0, \\ \left.\|u\|_{L^{\infty}\left((0, S), \dot{H}^{\mu}\left(\mathbb{R}^{n}\right)\right) \cap \bigcap_{j=1,2} L^{q_{j}}\left((0, S), \dot{B}_{r_{j}}^{\mu}\right.}\left(\mathbb{R}^{n}\right)\right) & \text { if } \mu>0 .\end{cases}
$$

Here, $\dot{H}^{\mu}\left(\mathbb{R}^{n}\right)$ and $\dot{B}_{r_{j} 2}^{\mu}\left(\mathbb{R}^{n}\right)$ denote the homogeneous Sobolev and Besov spaces, respectively. Since the propagator of the linear part of the first equation in (1.2) is written as $\exp ( \pm i \hbar \exp (-2 i \omega) s \Delta / 2 m)$, we assume $0 \leq \pm \omega \leq \pi / 2$ to define it as a pseudo-differential operator. We note that the scaling critical number of $p$ for (1.2) is $p\left(\mu_{0}\right):=1+4 /\left(n-2 \mu_{0}\right)$ when $a(\cdot)=1$. We put

$$
p_{1}\left(\mu_{0}\right):=1+\frac{4}{n-2 \mu_{0}} \cdot\left(1+\frac{4}{n-2 \mu_{0}} \cdot \frac{2 \mu_{0}}{n(1+\sigma)}\right)^{-1}
$$

for $\sigma \neq-1$.

Theorem 1.3. Let $n \geq 1, \lambda \in \mathbb{C}, 0 \leq \mu_{0}<n / 2$, and $1 \leq p \leq p\left(\mu_{0}\right)$. Let $\omega$ satisfies $0 \leq \pm \omega \leq \pi / 2$ and $\omega \neq-\pi / 2$. Assume $\mu_{0}<p$ if $p$ is not an odd number. There exist two admissible pairs $\left\{\left(q_{j}, r_{j}\right)\right\}_{j=1,2}$ with the following properties.

(1) (Local solutions.) For any $u_{0} \in H^{\mu_{0}}\left(\mathbb{R}^{n}\right)$, there exist $S>0$ with $S \leq S_{0}$ and a unique local solution $u$ of (1.2) in $X^{\mu_{0}}([0, S))$. Here, $S$ depends only on the norm $\left\|u_{0}\right\|_{\dot{H}^{\mu_{0}\left(\mathbb{R}^{n}\right)}}$ when $p<p\left(\mu_{0}\right)$, while $S$ depends on the profile of $u_{0}$ when $p=p\left(\mu_{0}\right)$. The solutions depend on the initial data continuously.

(2) (Small global solutions.) Assume that one of the following conditions from (i) to (vi) holds: (i) $\mu_{0}=0, p=p(0)$, (ii) $\mu_{0}>0, p=p\left(\mu_{0}\right), a_{1} \geq 0$, (iii) $1<p<p\left(\mu_{0}\right), a_{1}>0, \sigma<-1$, (iv) $1<p<p_{1}\left(\mu_{0}\right), a_{1}<0, \sigma>-1$, (v) $p_{1}\left(\mu_{0}\right)<p<p\left(\mu_{0}\right), a_{1}>0, \sigma>-1$, (vi) $\mu_{0}>0,1<p<p\left(\mu_{0}\right), a_{1}>0, \sigma=-1$. If $\left\|u_{0}\right\|_{\dot{H}^{\mu_{0}\left(\mathbb{R}^{n}\right)}}$ is sufficiently small, then the solution $u$ obtained in (1) is a global solution, namely, $S=S_{0}$. 
Remark 1.4. The results in Theorem 1.3 also hold for the gauge variant equation

$$
\pm i \frac{2 m}{\hbar} \partial_{s} u(s, x)+e^{-2 i \omega} \Delta u(s, x)-\lambda e^{-2 i \omega} \frac{a(s)^{2}}{w(s)}|u w|^{p}(s, x)=0,
$$

provided $\mu_{0}<p$ when $p$ is not an even number.

Remark 1.5. The result (2) in Theorem 1.3 especially shows that we always have small global solutions for $1<p<1+4 / n$ when $a(\cdot)$ is not a constant in the conditions (iii) and (vi). This result is much different from the case $a(\cdot)=1$ in the following sense. In the case $a(\cdot)=1$ and $\omega=0$, namely (1.3), some weighted spaces, for example, $(1+|x|)^{-1} L^{2}\left(\mathbb{R}^{n}\right)$, have been needed for global solutions for $1+2 / n<p<1+4 / n$ (see [13, 30, 35]). There exist blow-up solutions for small initial data for $1<p \leq 1+2 / n$ (see [20]). In the case $a(\cdot)=1$ and $\omega= \pm \pi / 4$, namely (1.4), there exist blow-up solutions for small initial data for $1<p \leq 1+2 / n$ (see [10, 18, [23, 36]).

When $\lambda \in \mathbb{R}$, we are able to use the conservation law (see Lemma 4.1, below) to show global solutions for large data in $H^{1}\left(\mathbb{R}^{n}\right)$. The global solutions were shown for the Schrödinger equation (1.3) in [14, Theorem 3.1], for the complex GinzburgLandau equation (1.5) in [17, Proposition 4.2]. Blow-up solutions for initial data with negative energy are obtained by the concavity of an auxiliary function, the virial identity, and the Heisenberg uncertainty principle. We refer to [5, Section 6.5] for (1.3), [1, Theorem 1.8] for (1.3) in the hyperbolic space, and [19, Theorem 5.3] for (1.4). We show the following results for global and blow-up solutions for (1.2).

Corollary 1.6. Let $\mu_{0}=0$ or $\mu_{0}=1$. Let $\lambda>0$. Let $1 \leq p<1+4 / n$ when $\mu_{0}=0$. Let $1 \leq p<1+4 /(n-2)$ and $a_{1}(p-1-4 / n) \geq 0$ when $\mu_{0}=1$. For any $u_{0} \in H^{\mu_{0}}\left(\mathbb{R}^{n}\right)$, the local solution $u$ given by (1) in Theorem 1.3 is a global solution.

Corollary 1.7. Let $\mu_{0}=1, \lambda<0, a_{1} \geq 0$ and $1 \leq p<1+4 / n$. Let $\omega=0$ or $\omega=\pi / 2$. For any $u_{0} \in H^{1}\left(\mathbb{R}^{n}\right)$, the local solution $u$ given by (1) in Theorem 1.3 is a global solution.

Corollary 1.8. Let $\mu_{0}=1$ and $\lambda<0$. Let $\omega \neq 0, \pi / 2$. Put $p_{0}:=2 /(\sin 2 \omega)^{2}-1$. Let $p_{0}<p \leq 1+4 /(n-2)$. Let $a_{1}(p-1-4 / n) \leq 0$ and $S_{0}=\infty$. For any $u_{0} \in H^{1}\left(\mathbb{R}^{n}\right)$ with negative energy

$$
\int_{\mathbb{R}^{n}} \frac{1}{2}\left|\nabla u_{0}(x)\right|^{2}+\frac{\lambda a_{0}^{2}\left|u_{0}(x)\right|^{p+1}}{p+1} d x<0,
$$

the solution u given by (1) in Theorem 1.3 blows up in finite time. 
Corollary 1.9. Let $\mu_{0}=1$ and $\lambda<0$. Let $\omega=0$ or $\omega=\pi / 2$. Let $1+4 / n \leq$ $p \leq 1+4 /(n-2)$. Let $a_{1} \leq 0$ and $S_{0}=\infty$. For any $u_{0} \in H^{1}\left(\mathbb{R}^{n}\right)$ which satisfies $\left\||x| u_{0}(x)\right\|_{L_{x}^{2}\left(\mathbb{R}^{n}\right)}<\infty$ and (1.7), the solution $u$ given by (1) in Theorem 1.3 blows up in finite time.

To prove the above corollaries, we use two dissipative properties. One is from the parabolic structure of the first equation in (1.2) when $0< \pm \omega<\pi / 2$. The other is from the scale function $a(\cdot)$ when $\partial_{t} a(0)=a_{1} \neq 0$. Even if the equation does not have the parabolic structure when $\omega=0, \pi / 2$, the latter is very effective to obtain the global solutions. This fact is expected from that the dissipative term $n \partial_{t} a \partial_{t} \phi / a$ in (2.11), below, appears as the weight function of $a(\cdot)$ and $w(\cdot)$ in (1.2). For example, the energy estimate (4.2), below, shows the dissipative property of the equation when $\lambda a_{1}(p-1-4 / n)>0$ by (4.5). There is a large literature on the Cauchy problem for (1.3) and (1.4) (see, for example, [5, 6, 7, 34, 37]). The properties of semilinear Schrödinger equations of the form $\left(i \partial_{t}+\Delta_{g}\right) u=|u|^{p-1} u$ have been studied on certain compact or noncompact Riemannian manifold $(M, g)$, where $\Delta_{g}$ is the Laplace-Beltrami operator on $(M, g)$. In the hyperbolic space $\mathbb{H}^{n}$, the dispersive effect on Schrödinger equations was considered in [1], and the global existence of solutions with finite energy has been shown in [21]. In the de Sitter spacetime, a dissipative effect on Schrödinger equations was shown in [27].

Throughout the paper, the notation $A \lesssim B$ denotes the inequality $A \leq C B$ for some constant $C>0$ which is not essential in our argument. For any real number $1 \leq r \leq \infty$, its conjugate number is denoted by $r^{\prime}$ with $1 / r+1 / r^{\prime}=1$. For $\mu \in \mathbb{R}$ and $1 \leq r, m \leq \infty$, we use the Lebesgue space $L^{r}\left(\mathbb{R}^{n}\right)$, the Sobolev space $H^{\mu, r}\left(\mathbb{R}^{n}\right)$, the Besov space $B_{r m}^{\mu}\left(\mathbb{R}^{n}\right)$, and their homogeneous spaces $\dot{H}^{\mu, r}\left(\mathbb{R}^{n}\right)$ and $\dot{B}_{r m}^{\mu}\left(\mathbb{R}^{n}\right)$ (see [3] for the definitions and properties).

\section{Derivation of the equation}

We derive the scale-function $a(\cdot)$ in (1.1), and the first equation in (1.2) in this section. To do it, we generalize the Einstein equation and the Robertson-Walker metric for general dimensions and complex line elements (see [4] and [8] for $n=3$ and real line elements). In the following, the Greek letters $\alpha, \beta, \gamma, \cdots$ run from 0 to $n$, the Latin letters $j, k, \ell, \cdots$ run from 1 to $n$. We use the Einstein rule for the sum of indices of tensors, for example, $T_{\alpha}^{\alpha}:=\sum_{\alpha=0}^{n} T_{\alpha}^{\alpha}$ and $T^{i}{ }_{i}:=\sum_{i=1}^{n} T_{i}^{i}$. For the coordinate $x=\left(x^{0}, \cdots, x^{n}\right)$ in $\mathbb{R}^{1+n}$, we define the volume element $d V=$ $\sum_{\sigma} \operatorname{sign}(\sigma) d x^{\sigma(0)} \cdots d x^{\sigma(n)}$, where $\sigma$ denotes the permutation of $\{0, \cdots, n\}$. We consider a bilinear symmetric complex-valued functional $\langle\cdot, \cdot\rangle$ on the vector space spanned by the vectors $\left\{\partial / \partial x^{\alpha}\right\}_{\alpha=0}^{n}$. We put $g_{\alpha \beta}:=\left\langle\partial / \partial x^{\alpha}, \partial / \partial x^{\beta}\right\rangle$. We denote by 
$\left(g_{\alpha \beta}\right)$ the matrix whose components are given by $g_{\alpha \beta}$. Put $g:=\operatorname{det}\left(g_{\alpha \beta}\right)$. Let $\left(g^{\alpha \beta}\right)$ be the inverse matrix of $\left(g_{\alpha \beta}\right)$. We define the line element $g_{\alpha \beta} d x^{\alpha} d x^{\beta}$. For the change of variables $y=y(x)$, we have $\sqrt{-g(y)} d V(y)=(\operatorname{sign} \operatorname{det}(\partial y / \partial x)) \sqrt{-g(x)} d V(x)$, where $g(y)$ denotes the determinant of $\left(g_{\alpha \beta}(y)\right)$ with $g_{\alpha \beta}(y):=\left\langle\partial / \partial y^{\alpha}, \partial / \partial y^{\beta}\right\rangle$, where we take the square root of $-g$ as $-\pi<\arg (-g) \leq \pi$. For any contravariant tensor $T^{\alpha}$, we denote its parallel displacement from $x$ to $x+d x$ by $\widetilde{T}^{\alpha}(x+d x):=$ $T^{\alpha}(x)-\Gamma_{\beta \gamma}^{\alpha}(x) T^{\beta}(x) d x^{\gamma}$, where $\Gamma_{\beta \gamma}^{\alpha}(x)$ denotes the proportional constant at $x$. We assume that $\Gamma_{\beta \gamma}^{\alpha}$ satisfies the symmetry condition $\Gamma_{\beta \gamma}^{\alpha}=\Gamma_{\gamma \beta}^{\alpha}$ and

$$
\left(g_{\alpha \beta} \widetilde{T}^{\alpha} \widetilde{T}^{\beta}\right)(x+d x)=\left(g_{\alpha \beta} T^{\alpha} T^{\beta}\right)(x)+O\left(\sum_{0 \leq \alpha \leq n}\left(d x^{\alpha}\right)^{2}\right)
$$

for any $T^{\alpha}$ and $d x$. Then we have the Christoffel symbol

$$
\Gamma_{\beta \gamma}^{\alpha}=\frac{1}{2} g^{\alpha \delta}\left(\partial_{\beta} g_{\delta \gamma}+\partial_{\gamma} g_{\beta \delta}-\partial_{\delta} g_{\beta \gamma}\right) .
$$

We define the covariant derivative $\nabla_{\beta}$ for $T^{\alpha}$ by

$$
\nabla_{\beta} T^{\alpha}:=\lim _{d x^{\beta} \rightarrow 0} \frac{T^{\alpha}\left(x+\widehat{d x^{\beta}}\right)-\widetilde{T}^{\alpha}\left(x+\widehat{d x^{\beta}}\right)}{d x^{\beta}}=\partial_{\beta} T^{\alpha}+\Gamma^{\alpha}{ }_{\beta \gamma} T^{\gamma},
$$

where $\widehat{d x^{\beta}}:=\left(0, \cdots, 0, d x^{\beta}, 0, \cdots, 0\right)$. In general, we define

$$
\begin{aligned}
\nabla_{\delta} T^{\alpha \beta \cdots}{ }_{\mu \nu \cdots:}:=\partial_{\delta} T^{\alpha \beta \cdots}{ }_{\mu \nu \cdots}+\Gamma^{\alpha}{ }_{\delta \varepsilon} T_{\mu \nu \cdots}^{\varepsilon \beta \cdots} & +\Gamma^{\beta}{ }_{\delta \varepsilon} T^{\alpha \varepsilon \cdots}{ }_{\mu \nu \cdots}+\cdots \\
& -\Gamma_{\delta \mu}^{\varepsilon} T^{\alpha \beta \cdots}{ }_{\varepsilon \nu \cdots}-\Gamma^{\varepsilon}{ }_{\delta \nu} T^{\alpha \beta \cdots}{ }_{\mu \varepsilon \cdots}-\cdots
\end{aligned}
$$

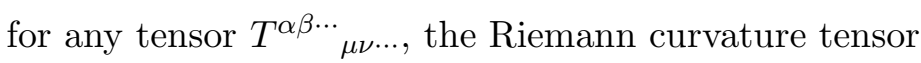

$$
R_{\alpha \beta \gamma}^{\delta}:=\partial_{\beta} \Gamma_{\alpha \gamma}^{\delta}-\partial_{\gamma} \Gamma_{\alpha \beta}^{\delta}+\Gamma_{\varepsilon \beta}^{\delta} \Gamma_{\alpha \gamma}^{\varepsilon}-\Gamma_{\varepsilon \gamma}^{\delta} \Gamma_{\alpha \beta}^{\varepsilon}
$$

which is derived from $R_{\alpha \beta \gamma}^{\delta} T^{\alpha}=\left(\nabla_{\beta} \nabla_{\gamma}-\nabla_{\gamma} \nabla_{\beta}\right) T^{\delta}$, the Ricci tensor $R_{\alpha \beta}:=$ $R^{\gamma}{ }_{\alpha \beta \gamma}$, the scalar curvature $R:=g^{\alpha \beta} R_{\alpha \beta}$. We define the Einstein tensor by $G_{\alpha \beta}:=$ $R_{\alpha \beta}-g_{\alpha \beta} R / 2$ which satisfies the Euler-Lagrange equation $G_{\alpha \beta}=0$ for the EinsteinHilbert action $\int_{\mathbb{R}^{1+n}} R \sqrt{-g} d x$. The change of upper and lower indices is done by $g_{\alpha \beta}$ and $g^{\alpha \beta}$, for example, $G_{\beta}^{\alpha}:=g^{\alpha \gamma} G_{\gamma \beta}$.

We put $r:=\left(\sum_{j=1}^{n}\left(x^{j}\right)^{2}\right)^{1 / 2}$ for any $\left(x^{1}, \cdots, x^{n}\right) \in \mathbb{R}^{n}$. We assume that the space is uniform and isotropic, and we consider the line element $(d \ell)^{2}:=-\left(d x^{0}\right)^{2}+$ $e^{h\left(x^{0}\right)} e^{f(r)} \sum_{j=1}^{n}\left(d x^{j}\right)^{2}$, where $h$ is a real-valued function and $f$ is a complex-valued function. This line element is uniform in the sense that for any two points $P$ and $Q$ in $\mathbb{R}^{n}$, the ratio of the coefficients $e^{h\left(x^{0}\right)} e^{f\left(r_{P}\right)} / e^{h\left(x^{0}\right)} e^{f\left(r_{Q}\right)}$ is independent of $x^{0}$. By a direct computation, we have $G^{0}{ }_{j}=G^{j}{ }_{0}=0$,

$$
G_{0}^{0}:=\frac{n-1}{2}\left(\frac{n}{4}\left(\partial_{0} h\right)^{2}-e^{-h-f}\left(f^{\prime \prime}+(n-1) \frac{f^{\prime}}{r}+\frac{n-2}{4}\left(f^{\prime}\right)^{2}\right)\right),
$$


and

$$
\begin{aligned}
G_{k}^{j}:=g_{k}^{j}\left(\frac{n-1}{2}\left(\partial_{0}^{2} h+\frac{n}{4}\left(\partial_{0} h\right)^{2}\right)-\right. & \left.\frac{n-2}{2} e^{-h-f}\left(f^{\prime \prime}+(n-2) \frac{f^{\prime}}{r}+\frac{n-3}{4}\left(f^{\prime}\right)^{2}\right)\right) \\
& +\frac{n-2}{2} e^{-h-f}\left(f^{\prime \prime}-\frac{f^{\prime}}{r}-\frac{\left(f^{\prime}\right)^{2}}{2}\right) \frac{x^{j} x^{k}}{r^{2}},
\end{aligned}
$$

where $f^{\prime}:=d f / d r$. Since the space is isotropic, the coefficient of $x^{j} x^{k}$ must vanish. So that, we assume that $f$ satisfies $f^{\prime \prime}-f^{\prime} / r-\left(f^{\prime}\right)^{2} / 2=0$, by which we obtain

$$
e^{f}=q^{2}\left(1+\frac{k^{2} r^{2}}{4}\right)^{-2}
$$

for constants $q(\neq 0), k \in \mathbb{C}$. For the stress-energy tensor $T^{\alpha}{ }_{\beta}:=\operatorname{diag}\left(\rho c^{2},-p, \cdots,-p\right)$ of the perfect fluid with the density $\rho$ and the pressure $p$, we define the $(1+n)$ dimensional Einstein equation

$$
G_{\beta}^{\alpha}-\Lambda g_{\beta}^{\alpha}=\kappa T_{\beta}^{\alpha},
$$

where $\Lambda \in \mathbb{R}$ is the cosmological constant, and the constant $\kappa$ is a positive real constant. We assume $\kappa c^{4}$ is a constant independent of $c$ (see Remark 2.1).

Remark 2.1. When $n=3, q, k \in \mathbb{R}$, the constant $\kappa$ is called the Einstein gravitational constant which is given by $\kappa=8 \pi \mathcal{G} / c^{4}$, where $\mathcal{G}$ is the Newton gravitational constant. For $n \geq 3, q, k \in \mathbb{C}$, the constant $\kappa$ is generalized to

$$
\kappa:=\frac{2(n-1) \pi^{n / 2} \mathcal{G}}{(n-2) \Gamma(n / 2) c^{4}} .
$$

Indeed, we define the equation of motion in the $(1+n)$-dimensional spacetime by $d^{2} x^{j} / d t^{2}+\partial_{j} \phi=0$, where $\phi:=-\mathcal{G} \rho *|x|^{2-n} /(n-2)$ is the gravitational potential and $\rho$ denotes the density function of mass. We denote the volume of the unit ball in $\mathbb{R}^{n}$ by $\omega_{n}:=2 \pi^{n / 2} / n \Gamma(n / 2)$. The potential $\rho$ satisfies the Poisson equation $\Delta \phi=$ $n \omega_{n} \mathcal{G} \rho$. The Euler-Lagrange equation for the Lagrangian $\left\{-g_{\alpha \beta}\left(d x^{\alpha} / d \tau\right)\left(d x^{\beta} / d \tau\right)\right\}^{1 / 2}$ yields the geodesic equation $d^{2} x^{\gamma} / d \tau^{2}+\Gamma^{\gamma}{ }_{\alpha \beta}\left(d x^{\alpha} / d \tau\right)\left(d x^{\beta} / d \tau\right)=0$, where $d \tau$ is the proper time defined by $(d \tau)^{2}=-(d \ell)^{2}$. We consider a line element $\eta_{\alpha \beta} d x^{\alpha} d x^{\beta}$ defined by $\left(\eta_{\alpha \beta}\right)=\operatorname{diag}(-1, C, \cdots, C)$ for a complex number $C$. We assume that $g_{\alpha \beta}$ is stationary $\partial_{0} g_{\alpha \beta}=0$ and symmetric $g_{\alpha \beta}=g_{\beta \alpha}$, and $g_{\alpha \beta}$ is weak in the sense that $\left|g_{\alpha \beta}-\eta_{\alpha \beta}\right|$ is sufficiently small. Moreover, we assume that the velocity of particles is very slow compared with the light speed $c$, and the pressure is almost zero, namely, $\left|d x^{j} / c d t\right| \ll 0$ and $p \ll 1$. Then we have $\Gamma_{00}^{0}=0, \Gamma_{00}^{j} \doteqdot-\partial_{j} g_{00} / 2 C$, and $T_{\alpha \beta} / c^{2} \doteqdot$ $\operatorname{diag}(-\rho, 0, \cdots, 0)$ by the definition of $\Gamma^{\alpha}{ }_{\beta \gamma}$ and $T_{\alpha \beta}$. We have $R_{00} \doteqdot-\partial_{j} \Gamma^{j}{ }_{00}$ by the definition of the Ricci tensor. We have $\left(d^{2} x^{\alpha} / d t^{2}\right) / c^{2}+\Gamma^{\alpha}{ }_{00} \doteqdot 0$ by the geodesic 
equation, which yields $\partial_{j}\left(\phi / c^{2}+g_{00} / 2 C\right) \doteqdot 0$ by the equation of motion and $\Gamma^{j} 00 \doteqdot$ $-\partial_{j} g_{00} / 2 C$. So that, we have $R_{00} \doteqdot \Delta g_{00} / 2 C \doteqdot-\Delta \phi / c^{2}=-n \omega_{n} \mathcal{G} \rho / c^{2}$. Since the Einstein equation (2.2) with $\Lambda=0$ is rewritten as $R_{\alpha \beta}=\kappa\left(T_{\alpha \beta}-g_{\alpha \beta} T /(n-1)\right)$, we obtain (2.3) from $R_{00}=\kappa\left(T_{00}-g_{00} T /(n-1)\right), T_{00} \doteqdot-\rho c^{2}$ and $T \doteqdot \rho c^{2}$.

We define a positive function $b\left(x^{0}\right):=e^{h\left(x^{0}\right) / 2}$. We put $\widetilde{\rho}:=\rho+\Lambda / \kappa c^{2}$, and $\widetilde{p}:=p-\Lambda / \kappa$. Then (2.2) is rewritten as $G^{\alpha}{ }_{\beta}=\kappa \cdot \operatorname{diag}\left(\widetilde{\rho} c^{2},-\widetilde{p}, \cdots,-\widetilde{p}\right)$, which shows that the cosmological constant $\Lambda>0$ is regarded as the energy which has positive density and negative pressure in the vacuum $\rho=p=0$ ("the dark energy" for $n=3)$. The equation $G_{0}^{0}=\kappa \widetilde{\rho} c^{2} g^{0}{ }_{0}$ is equivalent to

$$
\frac{n-1}{2}\left\{\left(\frac{\partial_{0} b}{b}\right)^{2}+\frac{k^{2}}{q^{2} b^{2}}\right\}=\frac{\kappa c^{2}}{n} \cdot \widetilde{\rho} .
$$

The equation $G^{j}{ }_{k}=-\kappa \widetilde{p} g^{j}{ }_{k}$ is equivalent to

$$
\frac{n-1}{2}\left\{\frac{2}{n-2} \cdot \frac{\partial_{0}^{2} b}{b}+\left(\frac{\partial_{0} b}{b}\right)^{2}+\frac{k^{2}}{q^{2} b^{2}}\right\}=-\frac{\kappa}{n-2} \cdot \widetilde{p},
$$

which is rewritten as the Raychaudhuri equation

$$
\frac{\partial_{0}^{2} b}{b}=-\frac{n-2}{n-1} \cdot \kappa\left(\frac{\widetilde{\rho} c^{2}}{n}+\frac{\widetilde{p}}{n-2}\right)
$$

by (2.4). Multiplying $b^{n}$ to the both sides in (2.4), and taking the derivative by $x^{0}$ variable, we have the conservation of mass

$$
\partial_{0}\left(\widetilde{\rho} c^{2} b^{n}\right)+\widetilde{p} \partial_{0} b^{n}=0
$$

For any real number $\sigma$, we assume the equation of state

$$
\widetilde{p}=\sigma \widetilde{\rho} c^{2}
$$

Then $b\left(x^{0}\right)$ must satisfy

$\frac{\partial_{0}^{2} b\left(x^{0}\right)}{b\left(x^{0}\right)}=-\frac{2}{n-1} \cdot \frac{n-2+n \sigma}{2 n} \cdot \kappa \widetilde{\rho} c^{2} \quad$ with $\quad \widetilde{\rho}=\frac{n-1}{2} \cdot \frac{n}{\kappa c^{2}} \cdot \frac{\partial_{0} b(0)^{2}}{b(0)^{2-n(1+\sigma)}} \cdot b\left(x^{0}\right)^{-n(1+\sigma)}$ by (2.6) and (2.7), which has the solution

$$
b\left(x^{0}\right):= \begin{cases}b(0)\left(1+\frac{n(1+\sigma) \partial_{0} b(0)}{2 b(0)} x^{0}\right)^{2 / n(1+\sigma)} & \text { if } \sigma \neq-1 \\ b(0) \exp \left(\frac{\partial_{0} b(0) x^{0}}{b(0)}\right) & \text { if } \sigma=-1\end{cases}
$$

By (2.4) and (2.9), we have $k=0$. 
For any $\lambda_{1} \in \mathbb{C}$ and any complex-valued $C^{2}$ function $\phi$ on $\mathbb{R}^{1+n}$, we define the Lagrangian

$$
L(\phi):=-\frac{1}{2} g^{\alpha \beta} \partial_{\alpha} \phi \partial_{\beta} \phi-\frac{1}{2}\left(\frac{m c}{\hbar}\right)^{2} \phi^{2}+\frac{\lambda_{1}}{p+1}|\phi|^{p-1} \phi^{2},
$$

and we apply the variational method to the action $\int_{\mathbb{R}^{1+n}} L(\phi) \sqrt{-g} d x$ for $\phi$. Then the Euler-Lagrange equation is given by

$$
\frac{1}{\sqrt{-g}} \partial_{\alpha}\left(\sqrt{-g} g^{\alpha \beta} \partial_{\beta} \phi\right)-\left(\frac{m c}{\hbar}\right)^{2} \phi+\lambda_{1}|\phi|^{p-1} \phi=0
$$

under the constraint condition $\arg \delta \phi=\arg \phi$. This is the equation of motion of massive scalar field described by a function $\phi$ with the mass $m$ and the potential $\lambda_{1}|\phi|^{p-1} \phi^{2} /(p+1)$.

We put $x^{0}:=$ ct. Then we have $a(t)=b\left(x^{0}\right)$ by (1.1) and (2.9). We put $q=e^{i \omega}$ for $-\pi / 2<\omega \leq \pi / 2$ in (2.1). Then the line element $d \ell$ is rewritten as $d \ell^{2}=-(c d t)^{2}+a(t)^{2} e^{i 2 \omega} \sum_{j=1}^{n}\left(d x^{j}\right)^{2}$. Then the field equation (2.10) is rewritten as

$$
-\frac{1}{c^{2}}\left(\partial_{t}^{2}+\frac{n \partial_{t} a}{a} \partial_{t}+\frac{m^{2} c^{4}}{\hbar^{2}}\right) \phi+\frac{1}{a^{2} e^{i 2 \omega}} \Delta \phi+\lambda_{1}|\phi|^{p-1} \phi=0 .
$$

We refer to [11], 26] and the references therein on energy solutions of (2.11) when $\omega=0$. We define a function $u$ by

$$
u\left(t, x^{1}, \cdots, x^{n}\right):=\phi(x)\left(\frac{a(t)}{a_{0}}\right)^{n / 2} \exp \left( \pm i \frac{m}{\hbar} c^{2} t\right) .
$$

Then the nonrelativistic limit $(c \rightarrow \infty)$ of (2.11) yields

$$
\pm i \frac{2 m}{\hbar} \partial_{t} u(t, x)+a(t)^{-2} e^{-2 i \omega} \Delta u(t, x)+\lambda_{1}\left(|u w|^{p-1} u\right)(t, x)=0 .
$$

By the change of variable $s:=\int_{0}^{t} a(\tau)^{-2} d \tau$, we obtain the first equation in (1.2) as $\lambda_{1}:=-\lambda e^{-2 i \omega}$. Since $u=u(t, \cdot)$ is a global solution if it exists on $\left[0, T_{0}\right)$, we say $u=u(s, \cdot)=u(t(s), \cdot)$ is a global solution if it exists on $\left[0, S_{0}\right)$.

Remark 2.2. In the above argument, we have derived the line element of the form

$$
-(c d t)^{2}+a(t)^{2} q^{2}\left(1+\frac{k^{2} r^{2}}{4}\right)^{-2} \sum_{j=1}^{n}\left(d x^{j}\right)^{2}
$$

for constants $q(\neq 0), k \in \mathbb{C}$ as the solution of (2.2). This is known as the RobertsonWalker metric when $n=3, q=1$ and $k^{2}=0, \pm 1$. Here, $k^{2}$ denotes the curvature of the space. We note that $a(\cdot)$ in (1.1) blows up in finite time when $a_{1}>0$ and $\sigma<-1$, which is called Big-Rip in cosmology. The case $\sigma=-1$ shows the exponential expansion of $a(\cdot)$ when $a_{1}>0$. The case $\sigma>-1$ shows the polynomial expansion of $a(\cdot)$ when $a_{1}>0$. These models are studied for the accelerating expansion of the universe when $n=3$. 
Remark 2.3. We have assumed the equation of state (2.8) to derive (2.9). Instead of this equation, let us assume $p=0, \rho \geq 0, \Lambda \in \mathbb{R}, k^{2} / q^{2} \in \mathbb{R}$. Then we have the $(1+n)$-dimensional version of the $(1+3)$-dimensional models of the universe as follows. By (2.4), $b(\cdot)$ must satisfy

$$
\left(\partial_{0} b\right)^{2}=R b^{2-n}+L b^{2}-\frac{k^{2}}{q^{2}}, \quad R:=\frac{2 \kappa \rho c^{2} b^{n}}{n(n-1)}, \quad L:=\frac{2 \Lambda}{n(n-1)},
$$

where $R$ is a constant by the conservation of mass. We have the following solutions for this differential equation. (1) If $\rho=\Lambda=0$, then $k^{2} / q^{2} \leq 0$ and $b\left(x^{0}\right)=b(0) \pm \sqrt{-k^{2} / q^{2}} x^{0}$. These solutions are the Minkowski model $(k=0)$, and the Milne model $\left(k^{2} / q^{2}<0\right)$ when $n=3$. (2) If $\rho=0$ and $\Lambda<0$, then $k^{2} / q^{2} \leq 0$ and $b\left(x^{0}\right)=\left|k^{2} / q^{2} L\right|^{1 / 2} \sin \left(|L|^{1 / 2} x^{0}\right)$. (3) If $\rho=0$ and $\Lambda>0$, then $b\left(x^{0}\right)=b(0) e^{ \pm L^{1 / 2} x^{0}}$ when $k^{2} / q^{2}=0, b\left(x^{0}\right)=\left(k^{2} / q^{2} L\right)^{1 / 2} \cosh \left(L^{1 / 2} x^{0}\right)$ when $k^{2} / q^{2}>0, b\left(x^{0}\right)=$ $\left|k^{2} / q^{2} L\right|^{1 / 2} \sinh \left(L^{1 / 2} x^{0}\right)$ when $k^{2} / q^{2}<0$. These solutions are the de Sitter models when $n=3$. (4) If $\rho>0$ and $\Lambda=0$, then $b\left(x^{0}\right)=\left(b(0)^{n / 2} \pm n R^{1 / 2} x^{0} / 2\right)^{2 / n}$ when $k^{2} / q^{2}=0$ (Einstein-de Sitter model for $n=3$ ), $b\left(x^{0}\right)=\left(q^{2} R(1-\cos 2 \theta) / 2 k^{2}\right)^{1 /(n-2)}$ with $\int_{0}^{\theta}((1-\cos 2 \theta) / 2)^{1 /(n-2)} d \theta=\left(k^{2} / q^{2}\right)^{1 / 2+1 /(n-2)} R^{-1 /(n-2)}(n-2) x^{0} / 2$ when $k^{2} / q^{2}>0, b\left(x^{0}\right)=\left(\left|q^{2}\right| R(\cosh 2 \theta-1) / 2\left|k^{2}\right|\right)^{1 /(n-2)}$ with $\int_{0}^{\theta}((\cosh 2 \theta-1) / 2)^{1 /(n-2)} d \theta$ $=\left|k^{2} / q^{2}\right|^{1 / 2+1 /(n-2)} R^{-1 /(n-2)}(n-2) x^{0} / 2$ when $k^{2} / q^{2}<0$. These solutions are the Friedmann model when $n=3$. (5) If $\rho>0$ and $\Lambda>0$, then $b\left(x^{0}\right)$ tends to infinity as $x^{0}$ tends to infinity for the cases (i) $k^{2} / q^{2} \leq 0$, or (ii) $k^{2} / q^{2}>0$ and $L>L_{0}$ (the Lemaitre model when $n=3$ ), or (iii) $k^{2} / q^{2}>0$ with $L=L_{0}:=$ $\left(k^{2} / 2 q^{2}\right)^{n /(n-2)} R^{-2 /(n-2)}$ and $b(0)>b_{*}:=(R / L)^{1 / n}$ (the Eddington-Lemaitre model when $n=3)$. For the case $k^{2} / q^{2}>0, L=L_{0}$ and $b(0)=b_{*}$, we have $b(\cdot)=b_{*}$ (the Einstein model when $n=3$ ). For the case $k^{2} / q^{2}>0, L=L_{0}$ and $b(0)<b_{*}$, $b(\cdot)$ tends to $b_{*}$ from below. For the case $k^{2} / q^{2}>0, L<L_{0}$ and $b(0)<b_{*}, b(\cdot)$ is bounded by $b_{*}$ from above and vanishes in finite time. For the case $k^{2} / q^{2}>0$, $L<L_{0}$ and $b(0)>b_{*}, b(\cdot)$ is bounded by $b_{*}$ from below and tends to infinity as $x^{0}$ tends to infinity.

Remark 2.4. Let us consider the relation between the line element $-(c d t)^{2}+$ $a(t)^{2} e^{i 2 \omega} \sum_{j=1}^{n}\left(d x^{j}\right)^{2}$ and transforms in spacetime. Let us consider two coordinates $x=\left(c t, x^{1}, \cdots, x^{n}\right)$ and $x_{*}=\left(c t_{*}, x_{*}^{1}, \cdots, x_{*}^{n}\right)$. We assume that the coordinate $x_{*}$ moves in the coordinate $x$ to infinity along $x^{1}$ axis at velocity $v \in \mathbb{R}$, and the origins in the coordinates coincide when $t=t_{*}=0$. By the spatial rotation in $x_{*}$, we may assume $x^{j}=x_{*}^{j}$ for $2 \leq j \leq n$. So that, it suffices to consider the relation between $\left(c t, x^{1}\right)$ and $\left(c t_{*}, x_{*}^{1}\right)$. Since we consider the uniform linear motion, $\left(\right.$ ct,$\left.x^{1}\right)$ and $\left(c t_{*}, x_{*}^{1}\right)$ has the transform ${ }^{T}\left(c_{*}, x_{*}^{1}\right)=A(v)^{T}\left(c t, x^{1}\right)$ by a matrix $A(v):=\left(\begin{array}{ll}a_{00} & a_{01} \\ a_{10} & a_{11}\end{array}\right)$ which is dependent on $v$, where ${ }^{T}\left(c t, x^{1}\right)$ de- 
notes the transpose of $\left(c t, x^{1}\right)$, and $a_{00}, a_{01}, a_{10}, a_{11} \in \mathbb{R}$. Since the point $\left(c t_{*}, 0\right)$ in $x_{*}$-coordinate is observed as $(c t, v t)$ in $x$-coordinate, and the point $(c t, 0)$ in $x$-coordinate is observed as $\left(c t_{*},-v t_{*}\right)$ in $x_{*}$-coordinate, we have $a_{10}=-a_{11} v / c$ and $a_{11}=a_{00}$. Since the coordinate $\left(c t, x^{1}\right)$ moves at velocity $-v$ in the coordinate $\left(c t_{*}, x_{*}^{1}\right)$ by the relativity, which means that $\left(c t,-x^{1}\right)$ moves at velocity $v$ in $\left(c t_{*},-x_{*}^{1}\right)$, we have ${ }^{T}\left(c t,-x^{1}\right)=A(v)^{T}\left(c t_{*},-x_{*}^{1}\right)$. So that, we have $a_{00}^{2}-a_{01} a_{10}=1$ since $\left(\begin{array}{c}c t_{*} \\ -x_{*}^{1}\end{array}\right)=\left(\begin{array}{cc}a_{00} & a_{01} \\ -a_{10} & -a_{11}\end{array}\right)\left(\begin{array}{c}c t \\ x^{1}\end{array}\right)$. Therefore, the components of $A(v)$ must satisfy

$$
a_{11}=a_{00}, \quad a_{10}=-\frac{v}{c} \cdot a_{00}, \quad a_{00}^{2}-a_{01} a_{10}=1 .
$$

Now, we assume the invariance of the line element, namely,

$$
-\left(c d t_{*}\right)^{2}+a\left(t_{*}\right)^{2} e^{i 2 \omega} \sum_{j=1}^{n}\left(d x_{*}^{j}\right)^{2}=-(c d t)^{2}+a(t)^{2} e^{i 2 \omega} \sum_{j=1}^{n}\left(d x^{j}\right)^{2}
$$

When $a(\cdot)=0$, we have $c d t_{*}=c d t$. Then we have $a_{00}=1$ and the Galilei transform $A(v)=\left(\begin{array}{cc}1 & 0 \\ -v / c & 1\end{array}\right)$ by (2.12). When $a(\cdot)=1$ and $\omega=\pi / 2$, we have $a_{00}^{2}+a_{10}^{2}=1$ and $a_{01}=-a_{10}$ by (2.13). Then we have the rotation of the spacetime $A(v)=$ $\left(1+(v / c)^{2}\right)^{-1 / 2}\left(\begin{array}{cc}1 & v / c \\ -v / c & 1\end{array}\right)$ by (2.12). When $a(\cdot)=1$ and $\omega=0$, we have $a_{00}^{2}-a_{10}^{2}=1$ and $a_{01}=a_{10}$ by (2.13). Then we have the Lorentz transform $A(v)=$ $\left(1-(v / c)^{2}\right)^{-1 / 2}\left(\begin{array}{cc}1 & -v / c \\ -v / c & 1\end{array}\right)$ by (2.12). When $a(\cdot)=1$ and $\omega \neq 0, \pi / 2$, we have $c d t_{*}=c d t, d x^{1}=d x_{*}^{1}, a_{00}=a_{11}=1$ and $a_{01}=a_{10}=0$ by (2.13), which requires $v=0$ by (2.12). Therefore, the rotational transform for the spatial variables $\left(x^{1}, \cdots, x^{n}\right)$ is only allowed in the case $\omega \neq 0, \pi / 2$.

Remark 2.5. We note that the line element with complex coefficients naturally unifies the rotational transform and the Lorentz transform as follows. Let us consider two coordinates $z=\left(z^{0}, z^{1}, \cdots, z^{n}\right) \in \mathbb{C}^{1+n}$ and $z_{*}=\left(z_{*}^{0}, z_{*}^{1}, \cdots, z_{*}^{n}\right) \in \mathbb{C}^{1+n}$ which satisfy the invariance of the line elements $\sum_{\alpha=0}^{n}\left(d z^{\alpha}\right)^{2}=\sum_{\alpha=0}^{n}\left(d z_{*}^{\alpha}\right)^{2}$. We assume $z^{j}=z_{*}^{j}$ for $2 \leq j \leq n$. For $\theta \in \mathbb{C}$, the transform $\left(\begin{array}{l}z_{*}^{0} \\ z_{*}^{1}\end{array}\right)=\left(\begin{array}{cc}\cos \theta & -\sin \theta \\ \sin \theta & \cos \theta\end{array}\right)\left(\begin{array}{l}z^{0} \\ z^{1}\end{array}\right)$ satisfies this invariance. For any fixed $-\pi / 2<\omega \leq \pi / 2$, let us consider the lines $z^{0}=i c t, z^{1}=x^{1} e^{i \omega}, z_{*}^{0}=i c t_{*}$ and $z_{*}^{1}=x_{*}^{1} e^{i \omega}$ in the complex plane $\mathbb{C}$, where $t, t_{*}, x^{1}, x_{*}^{1} \in \mathbb{R}$. Then we have the transform $\left(\begin{array}{c}c t_{*} \\ x_{*}^{1}\end{array}\right)=\left(\begin{array}{cc}\cos \theta & i e^{i \omega} \sin \theta \\ i e^{-i \omega} \sin \theta & \cos \theta\end{array}\right)\left(\begin{array}{l}c t \\ x^{1}\end{array}\right)$. So that, if $\omega=\pi / 2$ and $\theta \in \mathbb{R}$, then we have the rotational transform $\left(\begin{array}{c}c t_{*} \\ x_{*}^{1}\end{array}\right)=$ 


$$
\begin{aligned}
& \left(\begin{array}{cc}
\cos \theta & -\sin \theta \\
\sin \theta & \cos \theta
\end{array}\right)\left(\begin{array}{c}
c t \\
x^{1}
\end{array}\right) \text {. If } \omega=0 \text { and } i \theta \in \mathbb{R}, \text { then we have the Lorentz transform } \\
& \left(\begin{array}{c}
c t_{*} \\
x_{*}^{1}
\end{array}\right)=\left(\begin{array}{cc}
\cosh (i \theta) & \sinh (i \theta) \\
\sinh (i \theta) & \cosh (i \theta)
\end{array}\right)\left(\begin{array}{l}
c t \\
x^{1}
\end{array}\right) .
\end{aligned}
$$

\section{Proof of Theorem 1.3}

We define the operator $U_{ \pm}(s):=\exp ( \pm i \hbar \exp (-2 i \omega) s \Delta / 2 m)$. We put $V_{1}(\phi):=$ $\lambda|\phi|^{p-1} \phi$ and $f(s, u):=-i \hbar e^{-2 i \omega} a(s)^{2} w(s)^{-1} V_{1}((u w)(s)) / 2 m$. We regard the solution of the Cauchy problem of (1.2) as the fixed point of the integral equation given by

$$
u(s)=u(s, \cdot)=\Phi(u)(s):=U_{ \pm}(s) u_{0} \pm \int_{0}^{s} U_{ \pm}(s-\tau) f(\tau, u(\tau)) d \tau .
$$

We define $\left\{\left(q_{j}, r_{j}\right)\right\}_{j=0}^{2}$ by $1 / r_{j}=1 / 2-(p-1)\left(n-2 \mu_{0}\right) /(p+1) 2 n$ and $1 / r_{j}+2 / n q_{j}=$ $1 / 2$ for $j=0,1,2$. Then $\left\{\left(q_{j}, r_{j}\right)\right\}_{j=0}^{2}$ are admissible pairs by the condition on $p$. We define $q(\mu)$ by $1 / q(\mu)=1-(p-1)(n-2 \mu) / 4$ for $0 \leq \mu<n / 2$, and $r_{*}$ by $1 / r_{*}:=1 / r_{1}-\mu_{0} / n$. Then we have $1 / q\left(\mu_{0}\right):=1 / q_{0}^{\prime}-(p-1) / q_{1}-1 / q_{2}, r_{*}<\infty$ and $1 / r_{0}^{\prime}=(p-1) / r_{*}+1 / r_{2}$. We have

$$
\|\Phi(u)\|_{X^{0}([0, S))} \lesssim\left\|u_{0}\right\|_{L^{2}\left(\mathbb{R}^{n}\right)}+\|f(\cdot, u)\|_{L^{q^{\prime}}\left((0, S), L^{\left.r^{\prime}\left(\mathbb{R}^{n}\right)\right)}\right.}
$$

and

$$
\|\Phi(u)\|_{X^{\mu}([0, S))} \lesssim\left\|u_{0}\right\|_{\dot{H}^{\mu}\left(\mathbb{R}^{n}\right)}+\|f(\cdot, u)\|_{L^{q_{0}^{\prime}\left((0, S), \dot{B}_{r_{0}^{\prime}}^{\mu}\left(\mathbb{R}^{n}\right)\right)}}
$$

for any $\mu \in \mathbb{R}$ by the condition $0 \leq \pm \omega \leq \pi / 2$, the energy estimate for $\omega \neq 0$ and $\omega \neq \pi / 2$ (see, for example, [25, Lemma 2.1]), and the Strichartz estimate for $\omega=0$ and $\omega=\pi / 2$ (see [5], 15]). By Lemma 2.2 in [28], we have

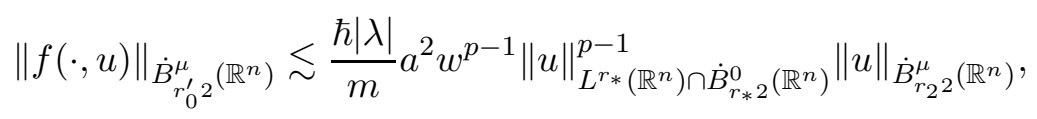

where the assumption $\mu<p$ is required if $p$ is not an odd number. By the embedding $\dot{B}_{r_{1} 2}^{\mu_{0}}\left(\mathbb{R}^{n}\right) \hookrightarrow L^{r_{*}}\left(\mathbb{R}^{n}\right) \cap \dot{B}_{r_{*} 2}^{0}\left(\mathbb{R}^{n}\right)$ and the Hölder inequality in time, we obtain

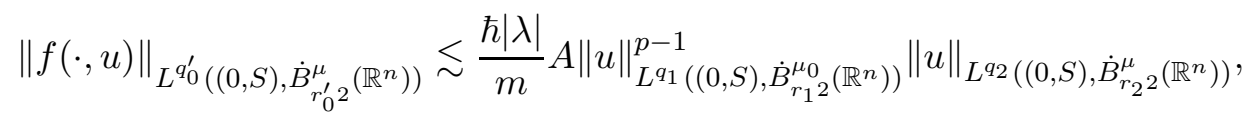

where we have put $A:=\left\|a(s)^{2} w(s)^{p-1}\right\|_{L_{s}^{q\left(\mu_{0}\right)}((0, S))}$. Analogously, we also have

$$
\|f(\cdot, u)\|_{L^{q_{0}^{\prime}}\left((0, S), L^{\left.r_{0}^{\prime}\left(\mathbb{R}^{n}\right)\right)}\right.} \lesssim \frac{\hbar|\lambda|}{m} A\|u\|_{L^{q_{1}}\left((0, S), \dot{B}_{r_{1} 2}^{\left.\mu_{0}\left(\mathbb{R}^{n}\right)\right)}\right.}^{p-1}\|u\|_{L^{q_{2}}\left((0, S), L^{\left.r_{2}\left(\mathbb{R}^{n}\right)\right)}\right.}
$$


and

$$
\begin{aligned}
& d(\Phi(u), \Phi(v)) \lesssim\|f(\cdot, u)-f(\cdot, v)\|_{L^{q_{0}^{\prime}\left((0, S), L^{r_{0}^{\prime}}\left(\mathbb{R}^{n}\right)\right)}}
\end{aligned}
$$

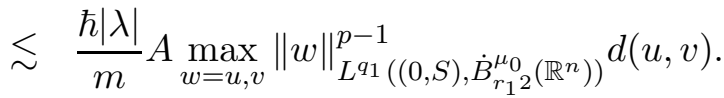

So that, $\Phi$ is a contraction mapping on the ball

$$
X\left(R_{0}, R_{\mu_{0}}\right):=\left\{u \in X^{\mu_{0}}([0, S)) ;\|u\|_{X^{\mu}([0, S))} \leq R_{\mu} \text { for } \mu=0, \mu_{0}\right\}
$$

with the metric $d$ if $R_{0}$ and $R_{\mu_{0}}$ satisfy

$$
R_{0} \geq 2 C_{0}\left\|u_{0}\right\|_{L^{2}\left(\mathbb{R}^{n}\right)}, \quad R_{\mu_{0}} \geq 2 C_{0}\left\|u_{0}\right\|_{\dot{H}^{\mu_{0}\left(\mathbb{R}^{n}\right)}}, \quad 2 C_{0} A R_{\mu_{0}}^{p-1} \leq 1
$$

for some constant $C_{0}>0$. We obtain the required fixed point by the Banach fixed point theorem.

When $p<p\left(\mu_{0}\right)$, we have $\lim _{S \searrow 0} A=0$ since $q\left(\mu_{0}\right) \neq \infty$. Thus, (3.4) is satisfied for any $u_{0} \in H^{\mu_{0}}$ and sufficiently small $S=S\left(\left\|u_{0}\right\|_{\dot{H}^{\mu_{0}\left(\mathbb{R}^{n}\right)}}\right)>0$. When $p=p\left(\mu_{0}\right)$, we have $\lim _{S \searrow 0} A=a_{0}^{2}$ since $q\left(\mu_{0}\right)=\infty$. So that, (3.4) requires the smallness of $\left\|u_{0}\right\|_{\dot{H}^{\mu_{0}\left(\mathbb{R}^{n}\right)}}$. To treat large $\left\|u_{0}\right\|_{\dot{H}^{\mu_{0}\left(\mathbb{R}^{n}\right)}}$, we put $u_{L}(s):=U_{ \pm}(s) u_{0}$ and show that the operator

$$
\widetilde{\Phi}\left(u_{N}\right)(s):= \pm \int_{0}^{s} U_{ \pm}(s-\tau) f\left(\tau,\left(u_{L}+u_{N}\right)(\tau)\right) d \tau
$$

is a contraction mapping on $X\left(R_{0}, R_{\mu_{0}}\right)$, where $R_{0} \geq \max _{j=1,2}\left\|u_{L}\right\|_{L^{q_{j}}\left((0, S), L^{r_{j}}\left(\mathbb{R}^{n}\right)\right)}$ and $R_{\mu_{0}} \geq \max _{j=1,2}\left\|u_{L}\right\|_{L^{q_{j}\left((0, S), \dot{B}_{r^{2}}\right.} \mu^{\left.\mu_{0}\left(\mathbb{R}^{n}\right)\right)}}$. Then the fixed point $u_{N}$ of $\widetilde{\Phi}$ gives the required solution $u$ by $u=u_{L}+u_{N}$. Similarly to the above argument, we have

$$
\begin{aligned}
& \left\|\widetilde{\Phi}\left(u_{N}\right)\right\|_{X^{\mu}([0, S))} \lesssim\left\|f\left(\cdot, u_{L}+u_{N}\right)\right\|_{L^{q_{0}^{\prime}}\left((0, S), \dot{B}_{r_{0}^{\prime}}^{\mu}\left(\mathbb{R}^{n}\right)\right)}
\end{aligned}
$$

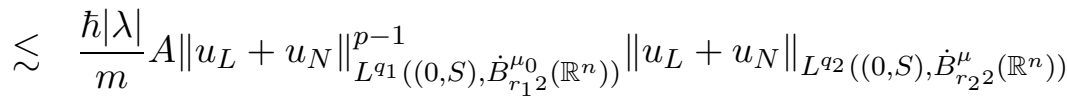

$$
\begin{aligned}
& \lesssim \frac{\hbar|\lambda|}{m} A\left(2 R_{\mu_{0}}\right)^{p-1} 2 R_{\mu}
\end{aligned}
$$

for any $\mu$ with $0<\mu \leq \mu_{0}$. We also have

$$
\left\|\widetilde{\Phi}\left(u_{N}\right)\right\|_{X^{0}([0, S))} \lesssim \frac{\hbar|\lambda|}{m} A\left(2 R_{\mu_{0}}\right)^{p-1} 2 R_{0}
$$

and

$$
d\left(\widetilde{\Phi}\left(u_{N}\right), \widetilde{\Phi}\left(v_{N}\right)\right) \lesssim \frac{\hbar|\lambda|}{m} A\left(2 R_{\mu_{0}}\right)^{p-1} d\left(u_{N}, v_{N}\right)
$$

for any $u_{N}, v_{N} \in X\left(R_{0}, R_{\mu_{0}}\right)$. Since $R_{\mu_{0}}$ can be taken sufficiently small when $S$ is sufficiently small by $q_{1} \neq \infty$ and $q_{2} \neq \infty, \widetilde{\Phi}$ is a contraction mapping if $S>0$ is 
sufficiently small. We note that $S$ depends on the profile of $u_{0}$ in addition to the norm $\left\|u_{0}\right\|_{\dot{H}^{\mu_{0}\left(\mathbb{R}^{n}\right)}}$.

The existence time $S$ of the solution $u$ is estimated from below by the condition (3.4). We recall $1 / q\left(\mu_{0}\right)=1-(p-1)\left(n-2 \mu_{0}\right) / 4$ and the change of variable $s=\int_{0}^{t} a(\tau)^{-2} d \tau$. When $p=p\left(\mu_{0}\right)$, we have

$$
A=a_{0}^{2} \cdot \begin{cases}1 & \text { if } a_{1} \geq 0, \\ \left(1+\frac{a_{1} n(1+\sigma) T}{2 a_{0}}\right)^{-\frac{8 \mu_{0}}{n(1+\sigma)\left(n-2 \mu_{0}\right)}} & \text { if } \sigma \neq-1 \text { and } a_{1}<0, \\ \exp \left(-\frac{4 \mu_{0} a_{1} T}{a_{0}\left(n-2 \mu_{0}\right)}\right) & \text { if } \sigma=-1 \text { and } a_{1}<0\end{cases}
$$

by a direct computation. So that, the solution $u$ is global for sufficiently small $u_{0}$ if (i) $\mu_{0}=0$, or (ii) $\mu_{0}>0$ and $a_{1} \geq 0$. When $p<p\left(\mu_{0}\right)$ and $\sigma \neq-1$, we have

$$
A^{q\left(\mu_{0}\right)}=a_{0}^{(p-1)\left(n-2 \mu_{0}\right) q\left(\mu_{0}\right) / 2} \cdot \begin{cases}\frac{2 a_{0}}{n a_{1}(1-\alpha)(1+\sigma)}\left(U^{1-\alpha}-1\right) & \text { if } a_{1} \neq 0 \text { and } \alpha \neq 1, \\ \frac{2 a_{0}}{n a_{1}(1+\sigma)} \log U & \text { if } a_{1} \neq 0 \text { and } \alpha=1, \\ T & \text { if } a_{1}=0,\end{cases}
$$

where we have put $U:=1+n a_{1}(1+\sigma) T / 2 a_{0}, \alpha:=2(p-1) \mu_{0} q\left(\mu_{0}\right) / n(1+\sigma)$. We note that $\alpha=1$ holds if and only if $p=p_{1}\left(\mu_{0}\right), \mu_{0} \neq 0$ and $\sigma>-1$ hold. So that, the solution $u$ is global for sufficiently small $u_{0}$ if (iii) $1<p<p\left(\mu_{0}\right), a_{1}>0, \sigma<-1$, or (iv) $1<p<p_{1}\left(\mu_{0}\right), a_{1}<0, \sigma>-1$, or (v) $p_{1}\left(\mu_{0}\right)<p<p\left(\mu_{0}\right), a_{1}>0, \sigma>-1$. When $p<p\left(\mu_{0}\right)$ and $\sigma=-1$, we have

$$
A^{q\left(\mu_{0}\right)}=a_{0}^{(p-1)\left(n-2 \mu_{0}\right) q\left(\mu_{0}\right) / 2} \cdot \begin{cases}\frac{1-\exp (-\beta T)}{\beta} & \text { if } \beta \neq 0, \\ T & \text { if } \beta=0,\end{cases}
$$

where we have put $\beta:=a_{1}(p-1) \mu_{0} q\left(\mu_{0}\right) / a_{0}$. So that, the solution $u$ is global for sufficiently small $u_{0}$ if $\beta>0$, where we note that $\beta>0$ holds if and only if $a_{1}>0$, $p>1$ and $\mu_{0}>0$ hold.

We prove the continuous dependence of solutions on initial data. Let $\left\{u_{j 0}\right\}_{j \geq 1}$ be a sequence of data which converges to $u_{0}$ in $H^{\mu_{0}}\left(\mathbb{R}^{n}\right)$, and let $\left\{u_{j}\right\}_{j \geq 1}$ be the solutions of (1.2) for $\left\{u_{j 0}\right\}_{j \geq 1}$. We use the equation

$$
\left(u-u_{j}\right)(s)=U_{ \pm}(s)\left(u_{0}-u_{j 0}\right) \pm \int_{0}^{s} U_{ \pm}(s-\tau)\left\{f(\tau, u(\tau))-f\left(\tau, u_{j}(\tau)\right)\right\} d \tau
$$

for $j \geq 1$, and the similar argument for (3.3) to obtain

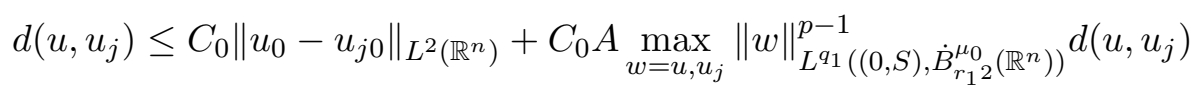

for some constant $C_{0}>0$. So that, we have $d\left(u, u_{j}\right) \rightarrow 0$ as $j \rightarrow \infty$ since $\lim _{S \searrow 0} A=$ 0 when $p<p\left(\mu_{0}\right)$. When $p=p\left(\mu_{0}\right)$, we use the argument starting from (3.5)). The nonlinear part $u_{j N}$ of $u_{j}$ is obtained with the property

$$
\max _{k=1,2}\left\|u_{j N}\right\|_{L^{q_{k}}\left((0, S), \dot{B}_{r_{k}}^{\mu_{0}}\left(\mathbb{R}^{n}\right)\right)} \leq \max _{k=1,2}\left\|u_{j L}\right\|_{L^{q_{k}}\left((0, S), \dot{B}_{r_{k}}^{\mu_{0}}\left(\mathbb{R}^{n}\right)\right)}
$$


under the condition

$$
2 C_{1} A\left(2 \max _{k=1,2}\left\|u_{j L}\right\|_{L^{q_{k}\left((0, S), \dot{B}_{r_{k}}{ }^{\mu_{0}}\left(\mathbb{R}^{n}\right)\right)}}\right)^{p-1} \leq 1
$$

for some constant $C_{1}>0$, where $u_{j L}:=U_{ \pm}(\cdot) u_{j 0}$. We are able to take $S>0$ uniformly for the existence time of $\left\{u_{j}\right\}_{j=1}^{\infty}$ since

$$
\begin{aligned}
& \max _{k=1,2}\left\|u_{j L}\right\|_{L^{q_{k}}\left((0, S), \dot{B}_{r_{k}}^{\mu_{0}}\left(\mathbb{R}^{n}\right)\right)} \leq \max _{k=1,2}\left\|u_{L}\right\|_{L^{q_{k}}\left((0, S), \dot{B}_{r_{k}}^{\mu_{0}}\left(\mathbb{R}^{n}\right)\right)} \\
& +C\left\|u_{0}-u_{j 0}\right\|_{\dot{H}^{\mu_{0}\left(\mathbb{R}^{n}\right)}}
\end{aligned}
$$

by the energy and Strichartz estimates, and $u_{j 0} \rightarrow u_{0}$ as $j \rightarrow \infty$. By (3.8) and (3.9), we have

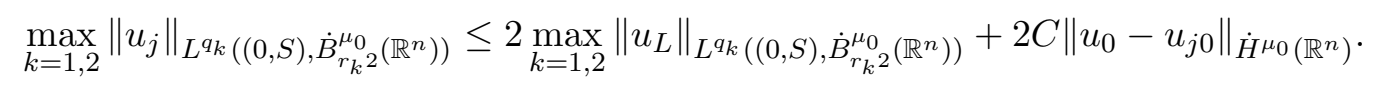

Since the right hand side tends to zero as $j \rightarrow \infty$ and $S \searrow 0$ by $q_{1} \neq \infty$ and $q_{2} \neq \infty$, we obtain $d\left(u, u_{j}\right) \rightarrow 0$ as $j \rightarrow \infty$ by (3.7).

\section{Proofs of Corollaries}

Let $V_{1}(z)$ be a complex-valued function for $z \in \mathbb{C}$. We consider the conservation law for the equation

$$
\pm i \frac{2 m}{\hbar} \partial_{s} u(s, x)+e^{-2 i \omega} \Delta u(s, x)-e^{-2 i \omega} \frac{a^{2}}{w}(s) V_{1}(u w)(s, x)=0
$$

for $0 \leq s<S$ and $x \in \mathbb{R}^{n}$.

Lemma 4.1. Let $V_{1}$ satisfy $\operatorname{Im}\left\{\bar{z} V_{1}(z)\right\}=0$ for any $z$ and its complex conjugate $\bar{z}$. For the solution $u$ of (4.1), the following results hold.

(1) The charge of u satisfies

$$
\begin{array}{r}
\frac{m}{\hbar} \int_{\mathbb{R}^{n}}|u(s, x)|^{2} d x \pm \sin 2 \omega \int_{0}^{s} \int_{\mathbb{R}^{n}}|\nabla u(\tau, x)|^{2}+\frac{a^{2}}{w}(\tau)\left(\bar{u} V_{1}(u w)\right)(\tau, x) d x d \tau \\
=\frac{m}{\hbar} \int_{\mathbb{R}^{n}}|u(0, x)|^{2} d x .
\end{array}
$$

(2) If there exists a function $V_{0}$ which satisfies $\partial_{s}\left\{V_{0}(v)\right\}=\operatorname{Re}\left\{\left(\partial_{s} \bar{v}\right) V_{1}(v)\right\}$ for any function $v$, then the energy of $u$ satisfies

$$
E(u)(s) \pm \frac{2 m \sin 2 \omega}{\hbar} \int_{0}^{s} \int_{\mathbb{R}^{n}}\left|\partial_{s} u(\tau, x)\right|^{2} d x d \tau+\int_{0}^{s} \int_{\mathbb{R}^{n}} I(\tau, x) d x d \tau=E(u)(0),
$$

where

$$
E(u)(s):=\int_{\mathbb{R}^{n}} \frac{1}{2}|\nabla u(s, x)|^{2}+\frac{a^{2}}{w^{2}}(s) V_{0}(u w)(s, x) d x
$$


and

$$
I(\tau, x):=\frac{n}{2 a_{0}^{n}} a(\tau)^{n+1} \frac{d a}{d s}(\tau)\left(\bar{u} w V_{1}(u w)-\frac{2(n+2)}{n} V_{0}(u w)\right)(\tau, x) .
$$

Proof. (1) Multiplying $\bar{u}$ to the equation (4.1) and taking its imaginary part, we obtain

$$
\pm \frac{m}{\hbar} \partial_{s}|u|^{2}+\nabla \cdot \operatorname{Im}\left(e^{-2 i \omega} \bar{u} \nabla u\right)+\left(|\nabla u|^{2}+\frac{a^{2}}{w} \bar{u} V_{1}(u w)\right) \sin 2 \omega=0 .
$$

We obtain the required result by the integration for $s$ and $x$.

(2) Multiplying $\partial_{s} \bar{u}$ to (4.1) and taking its real part, we have

$$
\partial_{s} e(u)-\nabla \cdot \operatorname{Re}\left(\partial_{s} \bar{u} \nabla u\right) \pm \frac{2 m \sin 2 \omega}{\hbar}\left|\partial_{s} u\right|^{2}+J=0
$$

where we have put $e(u):=|\nabla u|^{2} / 2+a^{2} w^{-2} V_{0}(u w)$ and

$$
J:=\frac{1}{2}\left\{a^{2} \partial_{s}\left(w^{-2}\right) \bar{u} w V_{1}(u w)-2 \partial_{s}\left(a^{2} w^{-2}\right) V_{0}(u w)\right\} .
$$

We note $I=J$ since $w=\left(a_{0} / a\right)^{n / 2}$. We obtain the required result by the integration for $s$ and $x$.

We put $V_{0}(v):=\lambda|v|^{p+1} /(p+1)$ and $V_{1}(v):=\lambda|v|^{p-1} v$ for $\lambda \in \mathbb{R}$. Then $V_{0}$ and $V_{1}$ satisfy the assumption in Lemma 4.1, and $I$ in the lemma is rewritten as

$$
I(\tau, x)=\frac{n}{2 a_{0}^{n}} a(\tau)^{n+1} \frac{d a}{d s}(\tau) V_{0}(u w)(\tau, x)\left(p-1-\frac{4}{n}\right) .
$$

Let us prove Corollary 1.6. Since the existence time of the solutions obtained by Theorem 1.3 is dependent only on the norms of the data when $p<p\left(\mu_{0}\right)$, it suffices to show the uniform bound of the solutions in $L^{2}\left(\mathbb{R}^{n}\right)$ when $\mu_{0}=0$, and $H^{1}\left(\mathbb{R}^{n}\right)$ when $\mu_{0}=1$. First, we show the case $\mu_{0}=0$. For any fixed $u_{0} \in L^{2}\left(\mathbb{R}^{n}\right)$, let $\left\{u_{j 0}\right\}_{j \geq 1} \subset H^{1}\left(\mathbb{R}^{n}\right)$ be a sequence of data which converges to $u_{0}$ in $L^{2}\left(\mathbb{R}^{n}\right)$, and let $\left\{u_{j}\right\}_{j \geq 1}$ be the solutions of (1.2) for $\left\{u_{j 0}\right\}_{j \geq 1}$ which are obtained by Theorem 1.3 . By the continuous dependence of the solutions on the data, there exists $S$ with $0<$ $S \leq S_{0}$ such that $u_{j}$ converges to $u$ in $L^{\infty}\left((0, S), L^{2}\left(\mathbb{R}^{n}\right)\right) \cap \bigcap_{k=1}^{2} L^{q_{k}}\left((0, S), L^{r_{k}}\left(\mathbb{R}^{n}\right)\right)$ as $j$ tends to infinity. By (1) in Lemma 4.1, $0 \leq \pm \omega \leq \pi / 2$ and $\lambda \geq 0$, we have $\int_{\mathbb{R}^{n}}\left|u_{j}(s, x)\right|^{2} d x \leq \int_{\mathbb{R}^{n}}\left|u_{j}(0, x)\right|^{2} d x$. Taking the limit of $j$, we have the required bound $\|u\|_{L^{\infty}\left((0, S), L^{2}\left(\mathbb{R}^{n}\right)\right)} \leq\left\|u_{0}\right\|_{L^{2}\left(\mathbb{R}^{n}\right)}$. Next, we show the case $\mu_{0}=1$. By (4.5), we have $I \geq 0$ if and only if $\lambda a_{1}(p-1-4 / n) \geq 0$. Thus, we have $\|\nabla u(s, \cdot)\|_{L^{2}\left(\mathbb{R}^{n}\right)}^{2} \leq$ $2 E(u)(s) \leq 2 E(u)(0)$ for $0 \leq s<S$ by $(2)$ in Lemma 4.1 $\lambda>0, a_{1}(p-1-$ $4 / n) \geq 0$ and $0 \leq \pm \omega \leq \pi / 2$. By (1) in Lemma 4.1, we also have $\|u(s, \cdot)\|_{L^{2}\left(\mathbb{R}^{n}\right)} \leq$ $\|u(0, \cdot)\|_{L^{2}\left(\mathbb{R}^{n}\right)}$ for $0 \leq s<S$. So that, $\|u\|_{L^{\infty}\left((0, S), H^{1}\left(\mathbb{R}^{n}\right)\right)}$ is uniformly bounded independent of $S$. 
Let us prove Corollary 1.7. By (4.5) and $\lambda a_{1}(p-1-4 / n) \geq 0$, we have $E(u)(s) \leq$ $E(u)(0)$ for $s \geq 0$. By the Gagliardo-Nirenberg inequality

$$
\|u\|_{L^{p+1}\left(\mathbb{R}^{n}\right)} \lesssim\|u\|_{L^{2}\left(\mathbb{R}^{n}\right)}^{1-n(p-1) / 2(p+1)}\|\nabla u\|_{L^{2}\left(\mathbb{R}^{n}\right)}^{n(p-1) / 2(p+1)},
$$

we have

$$
\begin{array}{r}
E(u)(s) \geq\left\{1-\frac{2 C|\lambda|\left(a^{2} w^{p-1}\right)(s)}{p+1}\|u(s)\|_{L^{2}\left(\mathbb{R}^{n}\right)}^{p+1-n(p-1) / 2}\|\nabla u(s)\|_{L^{2}\left(\mathbb{R}^{n}\right)}^{n(p-1) / 2-2}\right\} \\
\cdot \frac{\|\nabla u(s)\|_{L^{2}\left(\mathbb{R}^{n}\right)}^{2}}{2}
\end{array}
$$

for some constant $C>0$. By $\omega=0$ or $\omega=\pi / 2$, the conservation of charge $\|u\|_{L^{2}\left(\mathbb{R}^{n}\right)}=\left\|u_{0}\right\|_{L^{2}\left(\mathbb{R}^{n}\right)}$, and the condition $p<1+4 / n$, the inequality (4.6) shows that $\|\nabla u\|_{L^{2}\left(\mathbb{R}^{n}\right)}$ does not blow up before $S_{0}$. Namely, $u$ is a global solution.

Let us prove Corollary 1.8, By (4.5) and $\lambda a_{1}(p-1-4 / n) \geq 0$, we have $I \geq 0$. Thus, by (2) in Lemma 4.1, we have

$$
E(u)(s) \leq E(u)(0) \mp \frac{2 m \sin 2 \omega}{\hbar} \int_{0}^{s} \int_{\mathbb{R}^{n}}\left|\partial_{s} u(\tau, x)\right|^{2} d x d \tau .
$$

On the other hand, by (4.4) and $\overline{u w} V_{1}(u w)=(p+1) V_{0}(u w)$, we have

$$
\partial_{s} \int_{\mathbb{R}^{n}}|u(s, x)|^{2} d x \geq \mp \frac{\hbar \sin 2 \omega}{m}(p+1) E(u)(s) .
$$

We put $K(s):=\int_{0}^{s} \int_{\mathbb{R}^{n}}|u(\tau, x)|^{2} d x d \tau+K_{0}$ for a constant $K_{0}>0$ which is determined later. By (4.7) and (4.8), we have

$$
\partial_{s}^{2} K(s) \geq \mp \frac{\hbar \sin 2 \omega}{m}(p+1)\left\{E(u)(0) \mp \frac{2 m \sin 2 \omega}{\hbar} \int_{0}^{s} \int_{\mathbb{R}^{n}}\left|\partial_{s} u(\tau, x)\right|^{2} d x d \tau\right\} .
$$

Since $\partial_{s} K(s)$ satisfies

$$
\partial_{s} K(s)=\int_{\mathbb{R}^{n}}\left|u_{0}(x)\right|^{2} d x+2 \operatorname{Re} \int_{0}^{s} \int_{\mathbb{R}^{n}}\left(u \partial_{s} \bar{u}\right)(\tau, x) d x d \tau
$$

by the definition of $K(s)$, we have

$$
\begin{aligned}
\left(\partial_{s} K(s)\right)^{2} \leq\left(1+\frac{1}{\varepsilon}\right) & \left(\int_{\mathbb{R}^{n}}\left|u_{0}(x)\right|^{2} d x\right)^{2} \\
& +4(1+\varepsilon) \int_{0}^{s} \int_{\mathbb{R}^{n}}|u(\tau, x)|^{2} d x d \tau \int_{0}^{s} \int_{\mathbb{R}^{n}}\left|\partial_{s} u(\tau, x)\right|^{2} d x d \tau
\end{aligned}
$$

for any $\varepsilon>0$ by the Hölder inequality. So that, for any $\alpha$ with $\alpha>-1$, we have

$$
\begin{aligned}
\partial_{s}^{2} K(s) K(s)-(1+\alpha)\left(\partial_{s} K(s)\right)^{2} & \geq \mp \frac{\hbar \sin 2 \omega}{m}(p+1) E(u)(0) K_{0} \\
-(1+\alpha)\left(1+\frac{1}{\varepsilon}\right)\left(\int_{\mathbb{R}^{n}}\left|u_{0}(x)\right|^{2} d x\right)^{2}+2\left\{(\sin 2 \omega)^{2}(p+1)-2(1+\alpha)(1+\varepsilon)\right\} & \cdot \int_{0}^{s} \int_{\mathbb{R}^{n}}|u(\tau, x)|^{2} d x d \tau \int_{0}^{s} \int_{\mathbb{R}^{n}}\left|\partial_{s} u(\tau, x)\right|^{2} d x d \tau .
\end{aligned}
$$


Therefore, if $\omega \neq 0, \omega \neq \pi / 2, E(u)(0)<0, K_{0}$ is sufficiently large, $\alpha>0$ and $\varepsilon>0$ are sufficiently small, and $p>2 /(\sin 2 \omega)^{2}-1$, then we have $\partial_{s}^{2} K(s) K(s)-(1+$ $\alpha)\left(\partial_{s} K(s)\right)^{2}>0$. So that, we have

$$
\frac{1}{K(s)^{\alpha}}<\frac{K(0)-s \alpha\left\|u_{0}\right\|_{L^{2}\left(\mathbb{R}^{n}\right)}^{2}}{K(0)^{1+\alpha}} .
$$

since $\partial_{s}\left(\partial_{s} K / K^{1+\alpha}\right)=\left(\partial_{s}^{2} K K-(1+\alpha)\left(\partial_{s} K\right)^{2}\right) / K^{2+\alpha}>0$. This inequality shows $K(s)$ blows up at finite time $s=S_{1}:=K(0) / \alpha\left\|u_{0}\right\|_{L^{2}\left(\mathbb{R}^{n}\right)}^{2}$, which shows that there exists a sequence $\left\{s_{j}\right\}_{j \geq 1}$ which converges to $S_{1}$ such that $\int_{\mathbb{R}^{n}}\left|u\left(s_{j}, x\right)\right|^{2} d x$ tends to infinity as $j \rightarrow \infty$.

Let us prove Corollary 1.9, We use the virial identities $\partial_{s} \int_{\mathbb{R}^{n}}|x|^{2}|u(s, x)|^{2} d x=$ $\pm 2 \hbar e^{-2 i \omega} \operatorname{Im} \int_{\mathbb{R}^{n}} \bar{u} x \cdot \nabla u d x / m$, and

$$
\begin{aligned}
& \partial_{s}^{2} \int_{\mathbb{R}^{n}}|x|^{2} \mid\left.u(s, x)\right|^{2} d x \\
&=\left(\frac{\hbar}{m}\right)^{2}\left\{2 \int_{\mathbb{R}^{n}}|\nabla u(s, x)|^{2} d x+\frac{n(p-1) a(s)^{2}}{w(s)^{2}} \int_{\mathbb{R}^{n}} V_{0}(u w)(s, x) d x\right\},
\end{aligned}
$$

which are derived from the first equation in (1.2). Since we have

$$
\frac{a(s)^{2}}{w(s)^{2}} \int_{\mathbb{R}^{n}} V_{0}(u w)(s, x) d x=E(u)(s)-\frac{1}{2} \int_{\mathbb{R}^{n}}|\nabla u(s, x)|^{2} d x
$$

by the definition of $E(u)$, we have

$$
\begin{aligned}
\partial_{s}^{2} \int_{\mathbb{R}^{n}}|x|^{2}|u(s, x)|^{2} d x & \\
& \leq\left(\frac{\hbar}{m}\right)^{2}\left\{n(p-1) E(u)(s)-\frac{n}{2}\left(p-1-\frac{4}{n}\right) \int_{\mathbb{R}^{n}}|\nabla u(s, x)|^{2} d x\right\} .
\end{aligned}
$$

So that, we have

$$
\partial_{s}^{2} \int_{\mathbb{R}^{n}}|x|^{2}|u(s, x)|^{2} d x \leq \frac{\hbar^{2} n(p-1)}{m^{2}} E(u)(s)
$$

if $p \geq 1+4 / n$. Since $E(u)(s) \leq E(u)(0)$ by $a_{1} \lambda(p-1-4 / n) \geq 0$, the left hand side in (4.9) is bounded by a negative number from above if $E(u)(0)<0$. Therefore, there exists $S_{2}>0$ such that $\lim _{s}>_{2} \int_{\mathbb{R}^{n}}|x|^{2}|u(s, x)|^{2} d x=0$. By the Heisenberg uncertainty principle

$$
\|u\|_{L^{2}\left(\mathbb{R}^{n}\right)}^{2} \leq \frac{2}{n}\|\nabla u\|_{L^{2}\left(\mathbb{R}^{n}\right)}\||x| u\|_{L^{2}\left(\mathbb{R}^{n}\right)}
$$

and the conservation of charge $\|u(\cdot)\|_{L^{2}\left(\mathbb{R}^{n}\right)}=\left\|u_{0}\right\|_{L^{2}\left(\mathbb{R}^{n}\right)},\|\nabla u(s, \cdot)\|_{L^{2}\left(\mathbb{R}^{n}\right)}$ tends to infinity as $s$ tends to $S_{2}$. So that, the solution $u$ blows up in finite time. 


\section{References}

[1] V. Banica, The nonlinear Schrödinger equation on hyperbolic space, Comm. Partial Differential Equations 32 (2007), no. 10-12, 1643-1677.

[2] J. E. Barab, Nonexistence of asymptotically free solutions for a nonlinear Schrödinger equation, J. Math. Phys. 25 (1984), no. 11, 3270-3273.

[3] J. Bergh, J. Löfström, Interpolation spaces, An introduction, Grundlehren der Mathematischen Wissenschaften, No. 223, Springer-Verlag, Berlin-New York, 1976. x+207 pp.

[4] S. Carroll, Spacetime and geometry. An introduction to general relativity, Addison Wesley, San Francisco, CA, 2004. xiv+513 pp.

[5] T. Cazenave, Semilinear Schrödinger equations, Courant Lecture Notes in Mathematics, 10, New York University, Courant Institute of Mathematical Sciences, New York; American Mathematical Society, Providence, RI, 2003. xiv+323 pp.

[6] T. Cazenave, A. Haraux, An introduction to semilinear evolution equations, Oxford Lecture Series in Mathematics and its Applications, 13, The Clarendon Press, Oxford University Press, New York, 1998. xiv+186 pp.

[7] P. Cherrier, A. Milani, Linear and quasi-linear evolution equations in Hilbert spaces, Graduate Studies in Mathematics, 135, American Mathematical Society, Providence, RI, 2012. xviii +377 .

[8] R. d'Inverno, Introducing Einstein's relativity, The Clarendon Press, Oxford University Press, New York, 1992. xii+383 pp.

[9] G. B. Folland, A. Sitaram, The uncertainty principle: a mathematical survey, J. Fourier Anal. Appl. 3 (1997), no. 3, 207-238.

[10] H. Fujita, On the blowing up of solutions of the Cauchy problem for $u_{t}=\Delta u+u^{1+\alpha}$, J. Fac. Sci. Univ. Tokyo Sect. I 13 (1966), 109-124.

[11] A. Galstian, K. Yagdjian, Global solutions for semilinear KleinGordon equations in FLRW spacetimes, Nonlinear Analysis: Theory, Methods \& Applications 113 (2015), 339-356.

[12] J. Ginibre, T. Ozawa, Long range scattering for nonlinear Schrödinger and Hartree equations in space dimension $n \geq 2$, Comm. Math. Phys. 151 (1993), no. 3, 619-645.

[13] J. Ginibre, T. Ozawa, G. Velo, On the existence of the wave operators for a class of nonlinear Schrödinger equations, Ann. Inst. H. Poincaré Phys. Théor. 60 (1994), no. 2, 211-239.

[14] J. Ginibre, G. Velo, On a class of nonlinear Schrödinger equations. II. Scattering theory, general case, J. Funct. Anal. 32 (1979), no. 1, 33-71.

[15] J. Ginibre, G. Velo, Generalized Strichartz inequalities for the wave equation, J. Funct. Anal. 133 (1995), no. 1, 50-68. 
[16] J. Ginibre, G. Velo, The Cauchy problem in local spaces for the complex GinzburgLandau equation. I. Compactness methods, Phys. D 95 (1996), no. 3-4, 191-228.

[17] J. Ginibre, G. Velo, The Cauchy problem in local spaces for the complex GinzburgLandau equation. II. Contraction methods, Comm. Math. Phys. 187 (1997), no. 1, $45-79$.

[18] K. Hayakawa, On nonexistence of global solutions of some semilinear parabolic differential equations, Proc. Japan Acad. 49 (1973), 503-505.

[19] B. Hu, Blow-up theories for semilinear parabolic equations, Lecture Notes in Mathematics, 2018, Springer, Heidelberg, 2011. x+125 pp.

[20] M. Ikeda, Y. Wakasugi, Small-data blow-up of $L^{2}$-solution for the nonlinear Schrödinger equation without gauge invariance, Differential Integral Equations 26 (2013), no. 11-12, $1275-1285$.

[21] A. D. Ionescu, B. Pausader, G. Staffilani, On the global well-posedness of energy-critical Schrödinger equations in curved spaces, Anal. PDE 5 (2012), no. 4, 705-746.

[22] J. Kato, F. Pusateri, A new proof of long-range scattering for critical nonlinear Schrödinger equations, Differential Integral Equations 24 (2011), no. 9-10, 923-940.

[23] O. Kavian, Remarks on the large time behaviour of a nonlinear diffusion equation, Ann. Inst. H. Poincaré Anal. Non Linéaire 4 (1987), no. 5, 423-452.

[24] D. Li, Z. Dai, X. Liu, Long time behaviour for generalized complex Ginzburg-Landau equation, J. Math. Anal. Appl. 330 (2007), no. 2, 934-948.

[25] M. Nakamura, Small global solutions for nonlinear complex Ginzburg-Landau equations and nonlinear dissipative wave equations in Sobolev spaces, Reviews in Mathematical Physics 23 (2011), no. 8, 903-931.

[26] M. Nakamura, The Cauchy problem for semi-linear Klein-Gordon equations in de Sitter spacetime, J. Math. Anal. Appl. 410 (2014), no. 1, 445-454.

[27] M. Nakamura, On nonlinear Schrödinger equations derived from the nonrelativistic limit of nonlinear Klein-Gordon equations in de Sitter spacetime, Journal of Differential Equations 259 (2015), 3366-3388.

[28] M. Nakamura, T. Ozawa, Low energy scattering for nonlinear Schrödinger equations in fractional order Sobolev spaces, Rev. Math. Phys. 9 (1997), no. 3, 397-410.

[29] M. Nakamura, T. Ozawa, Small data scattering for nonlinear Schrödinger wave and Klein-Gordon equations, Ann. Sc. Norm. Super. Pisa Cl. Sci. (5) 1 (2002), no. 2, 435460 .

[30] K. Nakanishi, T. Ozawa, Remarks on scattering for nonlinear Schrödinger equations, NoDEA Nonlinear Differential Equations Appl. 9 (2002), no. 1, 45-68.

[31] W. van Saarloos, P. C. Hohenberg, Fronts, pulses, sources and sinks in generalized complex Ginzburg-Landau equations, Phys. D 56 (1992), no. 4, 303-367. 
[32] W. van Saarloos, P. C. Hohenberg, Erratum: "Fronts, pulses, sources and sinks in generalized complex Ginzburg-Landau equations," Phys. D 69 (1993), no. 1-2, 209.

[33] A. Schmid, A time dependent Ginzburg-Landau equation and its application to the problem of resistivity in the mixed state, Physik der kondensierten Materie 21, 5(1966), Issue $4,302-317$.

[34] T. Tao, Nonlinear dispersive equations. Local and global analysis, CBMS Regional Conference Series in Mathematics, 106, Published for the Conference Board of the Mathematical Sciences, Washington, DC; by the American Mathematical Society, Providence, RI, 2006. xvi+373.

[35] Y. Tsutsumi, K. Yajima, The asymptotic behavior of nonlinear Schrödinger equations, Bull. Amer. Math. Soc. (N.S.) 11 (1984), no. 1, 186-188.

[36] F. B. Weissler, Existence and nonexistence of global solutions for a semilinear heat equation, Israel J. Math. 38 (1981), no. 1-2, 29-40.

[37] S. Zheng, Nonlinear evolution equations, Chapman Hall/CRC Monographs and Surveys in Pure and Applied Mathematics, 133, Chapman \& Hall/CRC, Boca Raton, FL, 2004. xiv +287 . 